\title{
Association of dietary patterns with depressive symptoms in Chinese postmenopausal women
}

Kuan Liao ${ }^{1} \dagger$, Yeqing $\mathrm{Gu}^{1} \dagger$, Mingyue $\mathrm{Liu}^{1}$, Jingzhu Fu${ }^{1}$, Xiaotong Wang ${ }^{1}$, Guanlin Yang ${ }^{1}$, Qing Zhang ${ }^{2}$, $\mathrm{Li} \mathrm{Liu}^{2}$, Ge Meng ${ }^{1}$, Zhanxin Yao ${ }^{3}$, Hongmei Wu${ }^{1}$, Yang Xia ${ }^{1}$, Xue Bao ${ }^{1}$, Shunming Zhang ${ }^{1}$, Shaomei Sun ${ }^{2}$, Xing Wang ${ }^{2}$, Ming Zhou ${ }^{2}$, Huanli Jiao ${ }^{2}$, Qiyu Jia ${ }^{2}$, Kun Song ${ }^{2}$, Yuntang Wu ${ }^{1}$ and Kaijun Niu ${ }^{1,2,4,5 *}$

${ }^{1}$ Nutritional Epidemiology Institute and School of Public Health, Tianjin Medical University, Tianjin 300070, People's Republic of China

${ }^{2}$ Health Management Centre, Tianjin Medical University General Hospital, Tianjin 300070, People's Republic of China

${ }^{3}$ Institute of Environmental and Operational Medicine, Tianjin 300050, People's Republic of China

${ }^{4}$ Tianjin Key Laboratory of Environment, Nutrition and Public Health, Tianjin, People's Republic of China

${ }^{5}$ Center for International Collaborative Research on Environment, Nutrition and Public Health, Tianjin, People's Republic of China

(Submitted 5 January 2019 - Final revision received 20 June 2019 - Accepted 8 July 2019 - First published online 26 July 2019)

\section{Abstract}

Many components in diet have regulated oxidative stress, inflammatory reaction and even balance oestrogen levels. Because these factors are closely associated with depressive symptoms in postmenopausal women, it is considered that dietary factors are able to prevent and control depressive symptoms. On the other hand, a dietary pattern that considers the correlations and synergies between foods and nutrients is expected to have a greater impact on disease risk. The aim of the present study is to evaluate whether dietary patterns are associated with depressive symptoms in Chinese postmenopausal women. A cross-sectional study of 2051 postmenopausal women (mean age: 58.8 (sD 7.4) years) was conducted in Tianjin, China. Dietary consumption was assessed by a valid self-administered FFQ. Principal component analysis was used to derive three major dietary patterns: 'healthy', 'sweets' and 'traditional Tianjin' from eighty-eight food items. Depressive symptoms were assessed using the Zung Self-Rating Depression Scale, and cut-off point of 48 indicating serious depressive symptoms. The association between quartile of dietary patterns and depressive symptoms was assessed using multiple logistic regression analysis. The multivariable-adjusted OR of having depressive symptoms for increasing quartile of dietary patterns were as follows: healthy, 1.00, 0.79 (95\% CI 0.49, 1.28), 0.62 (95\% CI $0.37,1.04)$ and 0.57 (95\% CI 0.33, 0.97); sweets, 1.00, 0.75 (95\% CI 0.42, 1.3), 1.08 (95\% CI 0.64, 1.81) and 1.66 (95\% CI 1.03, 2.71); and traditional Tianjin, 1.00, 1.02 (95\% CI 0.58, 1.79), 0.96 (95\% CI 0.54, 1.71) and 2.53 (95\% CI 1.58, 4.16), respectively. The present study demonstrated that a healthy dietary pattern was inversely associated with depressive symptoms. On the contrary, greater adherence to sweets and traditional Tianjin dietary patterns was associated with a higher prevalence of depressive symptoms.

\section{Key words: Cross-sectional studies: Dietary patterns: Logistic models: Multivariate analyses: Depressive symptoms}

Globally, about 350 million people suffer from major depression $^{(1)}$. Depression, the most common of all the psychiatric disorders, caused high rates of morbidity and mortality and was reported as the second leading cause of years lived with disability ${ }^{(2)}$. Moreover, negative influences have been occurring in individuals with depressive symptoms such as attempting suicide ${ }^{(3)}$ and sleep disturbance ${ }^{(4)}$. Meanwhile, about $90 \%$ of individuals with mental disorders in China had never sought any type of professional help ${ }^{(5)}$. Women are nearly twice as likely, in contrast with men, to suffer from an episode of depression ${ }^{(6)}$, with an increased prevalence among those postmenopausal ${ }^{(7)}$. Thus, preventing high-risk population like postmenopausal women from depressive symptoms is urgent and significant.

Accumulated evidence suggested that dietary exposures are related to depressive symptoms with biological processes such as inflammation ${ }^{(8)}$ and oxidative processes $^{(9)}$. The most frequently investigated nutrients and food are vitamins B (including folate, riboflavin, pyridoxine and cobalamin), C and D, $n$-3 fatty acids, fish, fruits and vegetables ${ }^{(10)}$. Moreover, reduced

Abbreviation: MET, metabolic equivalent.

* Corresponding author: K. Niu, emails nkj0809@gmail.com, niukaijun@tmu.edu.cn

$\dagger$ These authors are co-first authors and contributed equally to this work. 
endogenous oestrogens are considered as a risk factor for postmenopausal depression ${ }^{(11)}$, and dietary phyto-oestrogens modulate sex hormone levels in postmenopausal women ${ }^{(12)}$.

Considering the correlations and synergies between foods and nutrients, it is difficult to identify associations for single items $^{(13)}$. A dietary pattern is a compositive variable that integrates consumption of several foods or food groups and is expected to have a greater impact on disease risk compared with any single nutrient or food ${ }^{(14)}$. Thus, we hypothesised that dietary patterns may have a potentially beneficial effect on the prevention of depressive symptoms in postmenopausal women. However, although a few studies have investigated the relationship between dietary patterns and depressive symptoms in middle-aged women ${ }^{(15,16)}$, only one Chinese Hong Kong study has investigated the relationship between dietary patterns and depressive symptoms in postmenopausal women $^{(17)}$. However, previous study indicated that food culture and dietary habits are substantially different among various regions in China ${ }^{(18)}$. We therefore designed a cross-sectional study to investigate how dietary patterns are related to depressive symptoms among postmenopausal women living in northern China.

\section{Materials and methods}

\section{Participants}

The Tianjin Chronic Low-grade Systemic Inflammation and Health (TCLSI-Health or TCLSIH) Cohort Study is an ongoing large prospective dynamic cohort study concerning association between chronic low-grade systemic inflammation and health status of a population living in Tianjin, China ${ }^{(19,20)}$. Female participants in this cross-sectional study were recruited annually during their health examinations at the Health Management Centre of Tianjin Medical University General Hospital.

This cross-sectional study used the baseline data from the TCLSIH during 2013-2016. Participants completed an examination including evaluation of anthropometric parameters and biochemical blood examination. And they were asked to finish a self-administered questionnaire consisting of demographic and socioeconomic characteristics, physical health status, lifestyle and health habits, depressive symptoms and FFQ.

During the study period, a total of 2621 participants with absence of a period for at least 1 year were sampled. We excluded participants who did not complete data collection on FFQ or depression scale $(n 516)$ or those with a history of CVD or cancer ( $n$ 54). Thus, 2051 participants (mean age 58.8, sD $7 \cdot 4$ years) were included in this analysis. The protocol of the present study has been approved by the Institutional Review Board of Tianjin Medical University, and each participant provided written informed consent for analysis of their data.

\section{Identification of dietary patterns}

The participants filled out a FFQ with guidance. The FFQ consists of 100 items, including seven frequency categories as follows: (1) almost never eat, (2) less than once per week, (3) once per week, (4) 2-3 times per week, (5) 4-6 times per week, (6) once per d and (7) twice or more per d for foods; and eight frequency categories are as follows: (1) almost never drink, (2) less than once per week, (3) once per week, (4) 2-3 times per week, (5) 4-6 times per week, (6) once per d, (7) twice or three times per $\mathrm{d}$ and (8) four or more times per $\mathrm{d}$ for beverages during the last month. Factor analysis was applied to find out major dietary patterns and factor loadings on all 100 food items and beverages. The reproducibility and validity of the questionnaire were assessed in a random sample of 150 participants living in Tianjin by comparing the data from repeat measure approximately 3 months apart and 4-d weighed dietary records (WDR). Spearman's rank correlation coefficient for energy intake between two FFQ was 0.68 ( $P<0 \cdot 0001)$, for food items (fruits, vegetables, fish, meat and beverages) ranged from 0.62 to 0.79 , for energy intake by the WDR and the FFQ was 0.49 and for nutrients (vitamins $\mathrm{C}$ and $\mathrm{E}$, polyunsaturated fats, saturated fats, carbohydrate and Ca) by the WDR and the FFQ ranged from 0.35 to 0.54 .

The FFQ was designed to measure food intake of the participants in the last month. Because the reproducibility and validity of the questionnaire have been assessed, the FFQ represents the long-term food intake of the participants. Moreover, seasonal food intake in the questionnaire includes the intake in the last month and in natural mature season.

Factor analysis (principal components analysis) was used to derive dietary patterns and to determine factor loadings for each of food and beverage subgroups (in $\mathrm{g} / \mathrm{d}$ ). Factors were rotated with varimax rotation to maintain uncorrelated factors and enhance interpretability. A combined evaluation of the eigenvalues, scree plot test and factor interpretability was used in determining the number of retained factors. The distinctive dietary patterns of the study population were well described by the three factors. Factors were named descriptively based on the food items showing high loading (absolute value $\geq 0.30$ ) with respect to each dietary pattern as follows: healthy dietary pattern (factor 1), sweets dietary pattern (factor 2) and traditional Tianjin dietary pattern (factor 3 ) (Table 1 ). In the present study, no component had a factor loading score $\leq-0 \cdot 30$. We calculated a factor score by summing the consumption from each food item weighted by its factor loading. A higher factor score indicates greater conformity to the dietary pattern. Variables unrelated to a given dietary pattern are weighted close to zero. For further analysis, factor scores were categorised into four equal groups using quartile cut-offs.

\section{Assessment of high depressive symptoms}

Depressive symptoms were evaluated by the Chinese version of the Zung Self-Rating Depression Scale. There were twenty items on the scale, which was defined as either positive or negative. Participants were required to grade on 1-4 points for each item. Summary scores of twenty items ranged from 20 to 80, with higher values indicating greater depressive symptoms. Since a previous study indicated that a cut-off point at 48 best matched clinical judgement ${ }^{(21)}$, and a cut-off point of 48 was also used to define depressive symptoms in other studies ${ }^{(22,23)}$, this cut-off point is used in the present study; scores higher than the cut-off indicate moderate depressive symptoms. 
Table 1. Factor loadings scores* of primary food groups of dietary patterns

\begin{tabular}{|c|c|c|c|c|c|}
\hline \multicolumn{2}{|l|}{ Healthy dietary pattern } & \multicolumn{2}{|l|}{ Sweets dietary pattern } & \multicolumn{2}{|c|}{ Traditional Tianjin dietary pattern } \\
\hline Food groups & $\begin{array}{l}\text { Factor } \\
\text { loadings }\end{array}$ & Food groups & $\begin{array}{l}\text { Factor } \\
\text { loadings }\end{array}$ & Food groups & $\begin{array}{l}\text { Factor } \\
\text { loadings }\end{array}$ \\
\hline Celery & 0.61 & Chinese cakes & 0.64 & Animal offal (exclude animal liver) & 0.58 \\
\hline Pumpkin, carrots & 0.56 & Ice cream & 0.63 & Preserved eggs & 0.56 \\
\hline Leafy vegetables & 0.54 & Sweets, candied fruits & 0.62 & Animal liver & 0.55 \\
\hline Potatoes (exclude sweet potatoes) & 0.53 & All kinds of cookies & 0.54 & Animal blood & 0.53 \\
\hline Eggplant & 0.53 & Pineapple & 0.53 & Instant noodles & 0.53 \\
\hline Cucumber & 0.52 & Strawberries, kiwi fruit, persimmon & 0.53 & Pork skin & 0.51 \\
\hline Chinese cabbage & 0.52 & Western-style pastry, cakes & 0.52 & Wonton & 0.47 \\
\hline Mushrooms & 0.52 & Watermelon & 0.52 & Steamed stuffed buns, dumplings & 0.39 \\
\hline White gourd & 0.52 & Peaches & 0.49 & Freshwater fish & 0.39 \\
\hline Tomatoes (include ketchup) & 0.51 & Grapes & 0.47 & Miscellaneous sauce noodles & 0.39 \\
\hline Radishes (exclude carrots) & 0.51 & Other fruits & 0.47 & Sea fish & 0.38 \\
\hline Bell peppers & 0.46 & Pickles & 0.42 & Sausages & 0.37 \\
\hline Sweet potatoes & 0.46 & Salted eggs & 0.39 & Seafood (shellfish, squid, shrimp) & 0.35 \\
\hline Raw vegetables & 0.45 & Pears & 0.39 & Pickles & 0.34 \\
\hline Apples & 0.45 & Bananas & 0.37 & Low-fat milk & 0.32 \\
\hline Ginger & 0.43 & Preserved bean curd & 0.36 & Bread & 0.32 \\
\hline Soyabean products & 0.43 & Sea grass & 0.36 & Salted eggs & 0.31 \\
\hline Garlic & 0.41 & Chinese chives & 0.34 & Poultry & 0.31 \\
\hline Lotus root & 0.40 & Nuts & 0.34 & Chillies & 0.30 \\
\hline Onion & 0.40 & Onion & 0.33 & & \\
\hline Coarse cereals & 0.38 & Apples & 0.31 & & \\
\hline Chillies & 0.38 & Oranges & 0.31 & & \\
\hline Eggs & 0.37 & Coffee & 0.30 & & \\
\hline Leeks & 0.36 & Walnuts & 0.30 & & \\
\hline Porridge & 0.35 & & & & \\
\hline Oranges & 0.34 & & & & \\
\hline Pears & 0.34 & & & & \\
\hline All types of beans & 0.32 & & & & \\
\hline
\end{tabular}

* The factor loading scores with an absolute value of more than 0.30 are shown.

\section{Assessment of other variables}

All participants received standardised physical examination at the Health Management Centre. Waist circumference was measured in standing position at the level of the umbilicus. Blood pressure was measured twice on the upper left arm in a sitting position and the average used for analysis. Fasting blood sugar was measured using the glucose oxidase method. TAG were measured using the enzymatic colorimetric method. LDL and HDL were measured with an autoanalyzer (Roche Cobas 8000 modular analyzer). The metabolic syndrome was defined according to the criteria of the American Heart Association Scientific Statement ${ }^{(24)}$.

Anthropometric variables (e.g. height and weight) were measured using a standard protocol. BMI was calculated as weight in kilograms divided by height in square metres. As for socioeconomic variables, educational level was defined by the question 'What is the highest degree you earned?' and was divided into two categories: <college graduate or $\geq$ college graduate. Marital status was classified as married or unmarried. The subjects were also classified as living alone or living with others. Occupation was classified as either senior officials and managers or professionals, while income was classified into two groups using the threshold of 10000 yuan per month. 'Visiting friends' status was assessed by asking the question 'Do you contact your friends and relatives often?' and classified as 'yes' or 'no'.

Information on the smoking ('never', 'former' and 'current smoking') and drinking ('never', 'former' and 'current drinking') status of the participants was obtained from a questionnaire survey. Physical activity in the most recent week was assessed using the short form of the International Physical Activity Questionnaire $^{(25)}$. The questionnaire asked whether subjects had performed any activities from the following categories during the previous week: walking; moderate activity (household activity or child care); and vigorous activity (running, swimming or other sports activities). Metabolic equivalent (MET) hours per week were calculated using the corresponding MET coefficients (3.3, 4. 0 and 8.0, respectively) according to the MET coefficient of activity $\times$ duration $(\mathrm{h} / \mathrm{d}) \times$ frequency $(\mathrm{d} /$ week $)$. Total physical activity levels were assessed in terms of weekly MET-h, which was calculated by combining separate hours for different activities. History of physical illness was evaluated on the basis of response ('yes' or 'no') to questions concerning a history of diseases (including liver diseases, gallstones, gastritis, chronic obstructive pulmonary diseases, pulmonary tuberculosis, gout, rheumatism, cataract, glaucoma, hearing disturbance, cervical spondylosis and lumbar spondylosis) and physician-diagnosed diseases (including diabetes, hypertension and the metabolic syndrome).

\section{Statistical analysis}

Descriptive data are presented as means with $95 \% \mathrm{CI}$ or as percentages and examined by ANOVA and $\chi^{2}$ test for categorical variables. Quartiles were categorised across the scores of each dietary pattern based on the distribution of the scores for all the participants and used for further analysis. Association between quartile categories of dietary pattern scores and 
Table 2. Baseline participant characteristics by depressed status (yes or no) according to the Self-Rating Depression Scale (SDS; cut-off point of 48) (Adjusted geometric mean values and $95 \%$ confidence intervals; percentages)

\begin{tabular}{|c|c|c|c|c|c|}
\hline & \multicolumn{4}{|c|}{ SDS score of 48} & \multirow[b]{3}{*}{$P^{*}$} \\
\hline & \multicolumn{2}{|c|}{ No $(n$ 1819) } & \multicolumn{2}{|c|}{ Yes $(n$ 232) } & \\
\hline & Mean & $95 \% \mathrm{Cl}$ & Mean & $95 \% \mathrm{Cl}$ & \\
\hline Age (years) & $58 \cdot 4$ & $58 \cdot 1,58.8$ & $56 \cdot 8$ & $55 \cdot 6,58$ & 0.01 \\
\hline $\mathrm{BMI}\left(\mathrm{kg} / \mathrm{m}^{2}\right)$ & 24.7 & $24.5,24.8$ & 24.2 & $23 \cdot 7,24 \cdot 7$ & 0.09 \\
\hline Waist circumference $(\mathrm{cm})$ & $82 \cdot 2$ & $81 \cdot 8,82 \cdot 6$ & $81 \cdot 3$ & $79 \cdot 8,82 \cdot 7$ & 0.24 \\
\hline $\mathrm{TC}(\mathrm{mmol} / \mathrm{l})$ & 5.36 & $5 \cdot 32,5 \cdot 4$ & 5.37 & $5 \cdot 22,5 \cdot 53$ & 0.90 \\
\hline LDL (mmol/l) & 3.16 & $3 \cdot 12,3 \cdot 2$ & 3.19 & $3.05,3.34$ & 0.69 \\
\hline $\mathrm{TAG}(\mathrm{mmol} / \mathrm{l})$ & 1.28 & $1.25,1.31$ & 1.15 & $1.06,1.25$ & 0.01 \\
\hline $\mathrm{HDL}(\mathrm{mmol} / \mathrm{l})$ & 1.48 & $1.46,1.31$ & 1.5 & $1.44,1.25$ & 0.46 \\
\hline $\mathrm{SBP}(\mathrm{mmHg})$ & $127 \cdot 4$ & $126 \cdot 5,128 \cdot 2$ & 123 & $120 \cdot 1,126 \cdot 1$ & $<0.01$ \\
\hline $\mathrm{DBP}(\mathrm{mmHg})$ & $77 \cdot 3$ & $76 \cdot 8,77 \cdot 8$ & 75.4 & $73 \cdot 7,77 \cdot 1$ & 0.04 \\
\hline $\mathrm{FBG}(\mathrm{mmol} / \mathrm{l})$ & $5 \cdot 2$ & $5 \cdot 16,5 \cdot 24$ & $5 \cdot 15$ & $5 \cdot 01,5 \cdot 3$ & 0.48 \\
\hline Physical activity (MET $\times \mathrm{h} /$ week) & $13 \cdot 4$ & $12 \cdot 6,14 \cdot 3$ & 8.49 & $6.73,10 \cdot 71$ & $<0.001$ \\
\hline Total energy intake $(\mathrm{kcal}+\mathrm{d})$ & 1931.5 & $1906,1957.4$ & $2030 \cdot 8$ & $1934.5,2131.9$ & 0.051 \\
\hline MetS (yes, \%) & & & & & 0.04 \\
\hline \multicolumn{6}{|l|}{ Smoking status (\%) } \\
\hline Smoker & \multicolumn{2}{|c|}{$2 \cdot 40$} & \multicolumn{2}{|c|}{0.20} & 0.77 \\
\hline Ex-smoker & \multicolumn{2}{|c|}{$1 \cdot 10$} & \multicolumn{2}{|c|}{$0 \cdot 10$} & 0.78 \\
\hline Non-smoker & \multicolumn{2}{|c|}{89.3} & \multicolumn{2}{|c|}{7.00} & 0.93 \\
\hline \multicolumn{6}{|l|}{ Drinker status (\%) } \\
\hline Every day & \multicolumn{2}{|c|}{1.40} & \multicolumn{2}{|c|}{0.10} & 0.47 \\
\hline Sometime & \multicolumn{2}{|c|}{31.4} & \multicolumn{2}{|c|}{2.50} & 0.63 \\
\hline Ex-drinker & \multicolumn{2}{|c|}{$6 \cdot 10$} & \multicolumn{2}{|c|}{0.50} & 0.78 \\
\hline Non-drinker & \multicolumn{2}{|c|}{$54 \cdot 2$} & \multicolumn{2}{|c|}{4.00} & 0.67 \\
\hline Marital status (married, \%) & \multicolumn{2}{|c|}{$92 \cdot 3$} & \multicolumn{2}{|c|}{$6 \cdot 8$} & 0.02 \\
\hline Living alone (yes, \%) & \multicolumn{2}{|c|}{6} & \multicolumn{2}{|c|}{0.3} & 0.45 \\
\hline Educational level ( $\geq$ college graduate, $\%$ ) & \multicolumn{2}{|c|}{$23 \cdot 8$} & \multicolumn{2}{|c|}{1.6} & 0.51 \\
\hline \multicolumn{6}{|l|}{ Employment status (\%) } \\
\hline Managers & \multicolumn{2}{|c|}{$25 \cdot 4$} & \multicolumn{2}{|c|}{2} & 1.00 \\
\hline Professionals & & & & & 0.79 \\
\hline Other & & & & & 0.88 \\
\hline Household income (>10 000 yuan, \%) & & & & & 0.98 \\
\hline Visiting friends (yes, \%) & & & & & 0.02 \\
\hline
\end{tabular}

TC, total cholesterol; SBP, systolic blood pressure; DBP, diastolic blood pressure; FBG, fasting blood glucose; MET, metabolic equivalents; MetS, metabolic syndrome.

${ }^{*}$ ANOVA or $\chi^{2}$ test.

† To convert kcal to kJ, multiply by $4 \cdot 184$.

depressive symptoms was examined using multiple logistic regression analysis. Depressed status was used as dependent variable, and factor score was used as independent variable. OR and $95 \%$ CI were calculated. A linear trend across increasing quartiles was tested using the median value of each quartile as a continuous variable based on linear regression. For model 1, the analysis was conducted without any adjustment; model 2 was adjusted for age and total energy intake; and model 3 was additionally adjusted to physical activity, smoking status, drinking status, education, job, income, marital status, visiting friends, living alone and metabolic symptom. Log transformation was used when the variables are not subject to normal distribution. $P$ values $<0.05$ were considered statistically significant and all tests presented were two tailed. All statistical analyses were performed using the Statistical Analysis System 9.1 edition for Windows (SAS Institute Inc.).

\section{Results}

After a varimax rotation, a factor analysis revealed three dietary patterns and the main factor loadings of each pattern (Table 1).
These three patterns explained $20.5 \%$ of the variance in dietary consumption (i.e. $8.9 \%$ for factor $1,6.3 \%$ for factor 2 and $5.3 \%$ for factor 3 ). According to their contributions to total variation, factor 1 was identified as a healthy dietary pattern characterised by a high consumption of vegetables, fruits and soyabean products; factor 2 was identified as a sweets dietary pattern typified by a greater consumption of ice cream, desserts and fruits; and factor 3 was defined as the traditional Tianjin dietary pattern characterised by a greater consumption of grain, milk, meat, animal blood, animal offal, sausages, preserved eggs, seafood and pickle products.

The participant characteristics according to their depressed status are presented in Table 2. Compared with participants without depressive symptoms, participants who had depressive symptoms tended to be younger $(P<0 \cdot 01)$ and had lower plasma concentrations of TAG $(P=0 \cdot 01)$, lower systolic blood pressure $(P<0.01)$, lower diastolic blood pressure $(P<0.05)$, decreased physical activity $(P<0.001)$, a lower prevalence of the metabolic syndrome $(P<0.05)$ and were less likely to be married $(P<0 \cdot 05)$.

Associations between dietary patterns and depressive symptoms are shown in Table 3. The healthy dietary pattern was 
Table 3. Adjusted relationships between quartiles of dietary pattern factor scores and depressive symptoms (Numbers of subjects; unadjusted and adjusted odds ratios and $95 \%$ confidence intervals)

\begin{tabular}{|c|c|c|c|c|c|c|c|c|}
\hline & \multicolumn{7}{|c|}{ Quartiles of dietary pattern factor score } & \multirow[b]{3}{*}{$P_{\text {for trend }}{ }^{*}$} \\
\hline & \multirow{2}{*}{$\begin{array}{c}\text { Level } 1 \\
\text { OR }\end{array}$} & \multicolumn{2}{|c|}{ Level 2} & \multicolumn{2}{|c|}{ Level 3} & \multicolumn{2}{|c|}{ Level 4} & \\
\hline & & OR & $95 \% \mathrm{Cl}$ & OR & $95 \% \mathrm{Cl}$ & OR & $95 \% \mathrm{Cl}$ & \\
\hline \multicolumn{9}{|l|}{ Healthy dietary pattern } \\
\hline No. of subjects & 513 & & 513 & & 512 & & 513 & \\
\hline No. of participants with high depressive symptoms & 69 & & 52 & & 58 & & 53 & \\
\hline Model 1 & 1.00 (reference) & 0.83 & $0.52,1.31$ & 0.73 & $0.45,1.17$ & 0.73 & $0.45,1.17$ & 0.18 \\
\hline Model $2 \dagger$ & 1.00 (reference) & 0.73 & $0.46,1 \cdot 18$ & 0.59 & $0.35,0.98$ & 0.56 & $0.33,0.94$ & 0.03 \\
\hline Model 3ł & 1.00 (reference) & 0.79 & $0.49,1.28$ & 0.62 & $0.37,1.04$ & 0.57 & $0.33,0.97$ & 0.03 \\
\hline \multicolumn{9}{|l|}{ Sweets dietary pattern } \\
\hline No. of subjects & 513 & & 513 & & 512 & & 513 & \\
\hline No. of participants with high depressive symptoms & 59 & & 45 & & 51 & & 77 & \\
\hline Model 1 & 1.00 (reference) & 0.76 & $0.44,1.32$ & $1 \cdot 11$ & $0.67,1.84$ & 1.83 & $1.16,2.93$ & $<0.001$ \\
\hline Model $2 \dagger$ & 1.00 (reference) & 0.74 & $0.43,1.28$ & 1.06 & $0.64,1.76$ & 1.63 & $1.09,2.77$ & $<0.01$ \\
\hline Model 3‡ & 1.00 (reference) & 0.75 & $0.42,1.3$ & 1.08 & $0.64,1.81$ & 1.66 & $1 \cdot 03,2 \cdot 71$ & $<0.01$ \\
\hline \multicolumn{9}{|l|}{ Traditional Tianjin dietary pattern } \\
\hline No. of subjects & 513 & & 513 & & 512 & & 513 & \\
\hline No. of participants with high depressive symptoms & 44 & & 51 & & 46 & & 91 & \\
\hline Model 1 & 1.00 (reference) & 1.04 & $0.60,1.82$ & 0.96 & $0.55,1.69$ & $2 \cdot 72$ & $1.71,4.43$ & $<0.0001$ \\
\hline Model $2 \dagger$ & 1.00 (reference) & 1.05 & $0.60,1.77$ & 0.97 & $0.55,1.72$ & $2 \cdot 60$ & $1.63,4.24$ & $<0.0001$ \\
\hline Model 3ł & 1.00 (reference) & 1.02 & $0.58,1.79$ & 0.96 & $0.54,1.71$ & 2.53 & $1.58,4.16$ & $<0.0001$ \\
\hline
\end{tabular}

* Multiple logistic regression analysis.

† Adjusted for age and total energy intake.

$\ddagger$ Adjusted for age, BMI, smoking status, drinking status, physical activity, marital status, total energy intake, household incomes, employment status, educational levels, visiting friends, living alone, metabolic syndrome.

inversely associated with prevalence of depressive symptoms. The OR for the extreme quartile was 0.57 (95\% CI $0.33,0.97$ ) $\left(P_{\text {for trend }}=0.03\right)$ after adjusting for all confounding factors. In contrast, participants with a high intake of sweets were more likely to report depressive symptoms. The OR across quartiles were 1.00 (reference), 0.75 (95\% CI 0.42, 1.3), 1.08 (95\% CI $0.64,1.81)$ and 1.66 (95\% CI 1.03, 2.71) after adjustments ( $\left.P_{\text {for trend }}<0 \cdot 01\right)$. Participants in the highest quartile of the traditional Tianjin dietary pattern had a $153 \%$ greater risk (OR 2.53; $95 \%$ CI $\left.1.58,4 \cdot 16 ; P_{\text {for trend }}<0.0001\right)$ of developing depressive symptoms than those in the lowest quartile after adjustments.

\section{Discussion}

In this cross-sectional study, we identified three dietary patterns, and all these dietary patterns were associated with depressive symptoms after adjustments for confounding variables. A healthy dietary pattern (vegetables, fruits and soyabean products) was associated with the decreased prevalence of depressive symptoms, whereas both greater adherence to sweets (ice cream, desserts and fruits) and traditional Tianjin dietary patterns (animal blood, animal offal, sausages and preserved eggs) were associated with a higher prevalence of depressive symptoms. The present study examines the relationship between dietary patterns and depressive symptoms in postmenopausal women.

In the present study, we have hypothesised that dietary patterns may have a potential correlation with depressive symptoms in postmenopausal women. A number of studies ${ }^{(26)}$ have investigated the relationship between dietary patterns and depressive symptoms in both men and women. A cross-sectional study among postmenopausal women living in Hong Kong China showed that dietary patterns featuring a low intake of processed foods (rich in refined cereals, sweets, preserved food, fried food and semi-lean animal meat) and/or a high intake of whole plant foods (rich in whole grains, fruits and vegetables), that were similar to the healthy dietary pattern in the present study, were associated with a reduced risk of depression ${ }^{(17)}$. To the best of our knowledge, no previous studies have assessed an association between dietary patterns and depressive symptoms in non-Chinese postmenopausal women. Only one Australian prospective study examined this association among middleaged women ${ }^{(16)}$. This prospective study suggested that a Mediterranean-style dietary pattern, that was similar to our healthy dietary pattern and was characterised by garlic, peppers, mushrooms, lettuce, onion or leeks, cucumber, pasta, rice, tomatoes, red wine, celery, other beans, avocado, bean sprouts and zucchini, had protective influences against the depressive symptoms ${ }^{(16)}$. However, no significant association was observed between the meat and processed meat dietary pattern that was similar to the traditional Tianjin dietary pattern in the present study and was characterised by pork, bacon, sausages and lamb and depressive symptoms. Moreover, we also found that a sweets dietary pattern, which had not been identified in other non-Chinese population, was positively associated with depressive symptoms. Thus, further studies are warranted to illustrate the associations between common and region-specific dietary patterns and depressive symptoms in postmenopausal women.

In the present study, the association between a healthy dietary pattern and depressive symptoms has been suggested by a number of plausible mechanisms in recent years. First, the high content of antioxidants such as carotenoids from vegetables and fruits may be an explanation for the protection ${ }^{(27)}$. Second, the potential protective effect of healthy dietary patterns 
could also come from large amounts of folate found in cruciferous vegetables, leafy vegetables and dried legumes ${ }^{(28)}$. Folate is hypothesised to protect brain function by decreasing homocysteine, which has a neurotoxic effect including impaired methylation, excitotoxicity, oxidative stress and hypoxia in the central nervous system ${ }^{(29)}$. Finally, Chinese are known to consume relatively high intake of soyabean and soya products ${ }^{(30)}$. Isoflavones, rich in soyabeans and soyabean products, are effective in reducing depressive and anxiety symptoms among postmenopausal women ${ }^{(31)}$ through selective $\beta$ oestrogenic receptor binding, and interactions with the dopaminergic, serotonergic and cholinergic systems, and brain regions crucial to higher cognitive function and $\operatorname{mood}^{(32)}$.

The present study also suggests that the sweets dietary pattern and traditional Tianjin dietary pattern are positively associated with depressive symptoms. The mechanisms that have been suggested to explain the positive association between the sweets dietary pattern and depressive symptoms are probably related to the high glycaemic load of the consumed items. The intake of a diet with a high glycaemic load has been associated in the short-term with rapid and immediate changes in serotonin levels and consequently with a relief of some psychological symptoms ${ }^{(33)}$. Increased inflammation and circulating cytokines have already been proven to be associated with depressive symptoms by extensive studies ${ }^{(34)}$. In fact, previous research shows that a higher intake of refined carbohydrates was associated with a higher level of C-reactive protein, a marker of proinflammatory cytokines ${ }^{(8)}$, which have already been proven to be possible mediators of known environmental risk factors in depression ${ }^{(34,35)}$. The traditional Tianjin dietary pattern characterised by preserved eggs, animal blood, animal offal, sausages and seafood contains a lot of fat. Also, consumption of a high-fat diet also leads to chronic systemic inflammation ${ }^{(36)}$. Another plausible mechanism relates to the consumption of a high$\mathrm{Pb}$-containing food, preserved eggs. Previous study suggested that dietary $\mathrm{Pb}$ exposure may increase the risk of mental health problems including depression ${ }^{(37)}$. This confirmed our previous studies that the sweets dietary pattern and traditional Tianjin dietary pattern were associated with high depressive symptoms among Chinese adults ${ }^{(38)}$.

Although this large population-based study considered many confounding factors, there are several limitations to our study. First, to confirm the level of depressive symptoms depends on not only total scores on the Self-Rating Depression Scale but also diagnostic interviews. Total scores do not correspond with a clinical diagnosis of depression but rather indicate the level of high depressive symptoms that may be of clinical relevance ${ }^{(39)}$. Therefore, further studies should conduct a standardised comprehensive structured diagnostic interview in order to measure the depressive symptoms more persuasively. Second, due to the nature of the self-reporting questionnaire, food intake may not be exact with recall bias. Finally, since this is a crosssectional study, reverse causation cannot examine in the present study. Depressive symptoms may have effect on dietary patterns that remains an alternative interpretation of the observed associations. Therefore, a prospective study or an intervention trial should be undertaken to confirm the existence of a relationship between the healthy dietary pattern and depressive symptoms.

\section{Conclusion}

In the present study, a higher score of the healthy dietary pattern characterised by vegetables, fruits, whole-grain food and soyabean products was inversely associated with depressive symptoms. On the contrary, adherence to the sweets dietary pattern characterised by ice cream, desserts and fruits and to the traditional Tianjin dietary pattern characterised by preserved eggs, animal blood, animal offal, sausages and seafood was positively associated with depressive symptoms. The findings suggest that dietary patterns appeared to be related to postmenopausal depressive symptoms. A long-term prospective study or randomised trials are required to clarify this causality.

\section{Acknowledgements}

We gratefully acknowledge the participants of the study and Tianjin Medical University General Hospital-Health Management Centre for the possibility to perform the study.

All data included in the present study are available upon request by contact with the corresponding author.

The present study was supported by grants from the National Natural Science Foundation of China (no. 81872611, 91746205, 81673166, 81372118, 81372467 and 81302422), the Key Technologies R\&D programme of Tianjin (Key Project: no. 11ZCGYSY05700, 12ZCZDSY20400, 13ZCZDSY20200, and 15YFYZSY00020), the National Science and Technology Support Programme (no. 2012BAI02B02), 2012 and 2016 Chinese Nutrition Society (CNS) Nutrition Research Foundation - DSM Research Fund (no. 2014-071, 2016-046 and 2016-023), the Technologies development programme of Beichen District of Tianjin (no. bcws2013-21, bcws2014-05 and 2015-SHGY-02), the technologies project of Tianjin Binhai New Area (no. 201302-04 and 2013-02-06), the Science Foundation of Tianjin Medical University (no. 2010KY28 and 2013KYQ24), the Key Laboratory of Public Health Safety (Fudan University), Ministry of Education (no. GW2014-5) and the National Training Programmes of Innovation and Entrepreneurship for Undergraduates (no. 201510062013), China.

K. L. and Y. G. analysed data and wrote the manuscript. M. L., J. F., X. W., G. Y., Q. Z., L. L., G. M. and Z. Y. contributed to the discussion and edited the manuscript. H. W., Y. X., X. B., S. Z., S. S., X. W., M. Z. and H. J. contributed to collect the data and interpreted the results. Q. J. and K. S. revised the manuscript for important intellectual contents. Y. W. and K. N. designed the study, reviewed and edited the manuscript. K. N. had full access to all the data and took responsibility for the integrity of the data.

The authors declare that there are no conflicts of interest regarding the publication of this paper.

\section{References}

1. World Health Organization (2016) Depression fact sheet. http://who.int/mediacentre/factsheets/fs369/en/

2. Ferrari AJ, Charlson FJ, Norman RE, et al. (2013) Burden of depressive disorders by country, sex, age, and year: findings from the global burden of disease study 2010. PLoS Med 10, e1001547. 
3. Chen Y, Bennett D, Clarke R, et al. (2017) Patterns and correlates of major depression in Chinese adults: a cross-sectional study of 0.5 million men and women. Psychol Med 47, 958-970.

4. Seow LS, Subramaniam M, Abdin E, et al. (2016) Sleep disturbance among people with major depressive disorders (MDD) in Singapore. J Ment Health 25, 492-499.

5. Phillips MR, Zhang J, Shi Q, et al. (2009) Prevalence, treatment, and associated disability of mental disorders in four provinces in China during 2001-05: an epidemiological survey. Lancet 373, 2041-2053.

6. Kessler RC, McGonagle KA, Swartz M, et al. (1993) Sex and depression in the National Comorbidity Survey. I: lifetime prevalence, chronicity and recurrence. J Affect Disord 29, 85-96.

7. Sassarini DJ (2016) Depression in midlife women. Maturitas 94, 149-154.

8. Liu S, Manson JE, Buring JE, et al. (2002) Relation between a diet with a high glycemic load and plasma concentrations of highsensitivity C-reactive protein in middle-aged women. Am J Clin Nutr 75, 492-498.

9. Black CN, Penninx BW, Bot M, et al. (2016) Oxidative stress, anti-oxidants and the cross-sectional and longitudinal association with depressive symptoms: results from the CARDIA study. Transl Psychiatry 6, e743.

10. Murakami K \& Sasaki S (2010) Dietary intake and depressive symptoms: a systematic review of observational studies. Mol Nutr Food Res 54, 471-488.

11. Georgakis MK, Thomopoulos TP, Diamantaras AA, et al. (2016) Association of age at menopause and duration of reproductive period with depression after menopause: a systematic review and meta-analysis. JAMA Psychiatry 73, 139-149.

12. Low YL, Dunning AM, Dowsett M, et al. (2007) Phytoestrogen exposure is associated with circulating sex hormone levels in postmenopausal women and interact with ESR1 and NR1I2 gene variants. Cancer Epidemiol Biomarkers Prev 16, 1009-1016.

13. Hodge A \& Bassett J (2016) What can we learn from dietary pattern analysis? Public Health Nutr 19, 191-194.

14. Hu FB (2002) Dietary pattern analysis: a new direction in nutritional epidemiology. Curr Opin Lipidol 13, 3-9.

15. Mihrshahi S, Dobson AJ \& Mishra GD (2015) Fruit and vegetable consumption and prevalence and incidence of depressive symptoms in mid-age women: results from the Australian longitudinal study on women's health. Eur J Clin Nutr 69, 585-591.

16. Rienks J, Dobson AJ \& Mishra GD (2013) Mediterranean dietary pattern and prevalence and incidence of depressive symptoms in mid-aged women: results from a large community-based prospective study. Eur J Clin Nutr 67, 75-82.

17. Liu ZM, Ho SC, Xie YJ, et al. (2016) Associations between dietary patterns and psychological factors: a cross-sectional study among Chinese postmenopausal women. Menopause 23, 1294-1302.

18. Song F \& Cho MS (2017) Geography of food consumption patterns between South and North China. Foods 6, 34.

19. Song K, Du H, Zhang Q, et al. (2014) Serum immunoglobulin $\mathrm{M}$ concentration is positively related to metabolic syndrome in an adult population: Tianjin Chronic LowGrade Systemic Inflammation and Health (TCLSIH) Cohort Study. PLOS ONE 9, e88701.

20. Sun S, Wu H, Zhang Q, et al. (2014) Subnormal peripheral blood leukocyte counts are related to the lowest prevalence and incidence of metabolic syndrome: Tianjin Chronic Lowgrade Systemic Inflammation and Health Cohort Study. Mediators Inflamm 2014, 412386.
21. Gregory RJ (2006) The Zung Self-rating Depression Scale as a potential screening tool for use with Eskimos. Psychiatric Services 45, 573-575.

22. Su Q, Yu B, He H, et al. (2016) Nut consumption is associated with depressive symptoms among Chinese adults. Depress Anxiety 33, 1065-1072.

23. Aoki A, Nagate M, Utsumi K, et al. (2012) Can we determine depressive conditions on the basis of somatic symptoms? A cross-sectional study of depressive conditions among Japanese patients at a university hospital general medicine clinic. Intern Med 51, 1335-1340.

24. Alberti KG, Eckel RH, Grundy SM et al. (2009) Harmonizing the metabolic syndrome: a joint interim statement of the International Diabetes Federation Task Force on Epidemiology and Prevention; National Heart, Lung, and Blood Institute; American Heart Association; World Heart Federation; International Atherosclerosis Society; and International Association for the Study of Obesity. Circulation 120, 1640-1645.

25. Craig CL, Marshall AL, Sjostrom M et al. (2003) International physical activity questionnaire: 12 -country reliability and validity. Med Sci Sports Exerc 35, 1381-1395.

26. Martinez-Gonzalez MA \& Sanchez-Villegas A (2016) Food patterns and the prevention of depression. Proc Nutr Soc $\mathbf{7 5}$, 139-146.

27. Beydoun MA, Beydoun HA, Boueiz A et al. (2013) Antioxidant status and its association with elevated depressive symptoms among US adults: National Health and Nutrition Examination Surveys 2005-6. Br J Nutr 109, 1714-1729.

28. Akbaraly TN, Brunner EJ, Ferrie JE et al. (2009) Dietary pattern and depressive symptoms in middle age. Br J Psychiatry $\mathbf{1 9 5}$, 408-413.

29. Bottiglieri T (2005) Homocysteine and folate metabolism in depression. Prog Neuropsychopharmacol Biol Psychiatry 29, 1103-1112.

30. Mei J, Yeung SS \& Kung AW (2001) High dietary phytoestrogen intake is associated with higher bone mineral density in postmenopausal but not premenopausal women. I Clin Endocrinol Metab 86, 5217-5221.

31. Lipovac M, Chedraui $P$, Gruenhut $C$ et al. (2010) Improvement of postmenopausal depressive and anxiety symptoms after treatment with isoflavones derived from red clover extracts. Maturitas 65, 258-261.

32. Craig MC \& Murphy DG (2007) Estrogen: effects on normal brain function and neuropsychiatric disorders. Climacteric 10 Suppl 2, 97-104

33. Murakami K, Sasaki S, Takahashi Y et al. (2008) Dietary glycemic index is associated with decreased premenstrual symptoms in young Japanese women. Nutrition 24, 554-561.

34. Berk M, Williams LJ, Jacka FN et al. (2013) So depression is an inflammatory disease, but where does the inflammation come from? BMC Med 11, 200.

35. Miller AH, Maletic V \& Raison CL (2009) Inflammation and its discontents: the role of cytokines in the pathophysiology of major depression. Biol Psychiatry 65, 732-741.

36. Schachter J, Martel J, Lin CS et al. (2018) Effects of obesity on depression: a role for inflammation and the gut microbiota. Brain Behav Immun 69, 1-8.

37. Yu B, Shao J, Yu F et al. (2017) Consumption of preserved egg, a high-lead-containing food, is strongly associated with depressive symptoms in Chinese adults. Br J Nutr 118, 737-742.

38. Xia Y, Wang N, Yu B et al. (2017) Dietary patterns are associated with depressive symptoms among Chinese adults: a casecontrol study with propensity score matching. Eur J Nutr 56, 2577-2587.

39. Zung WW (1965) A self-rating depression scale. Arch Gen Psychiatry 12, 63-70. 